\title{
In-to-out body Path Loss for Wireless Radio Frequency Capsule Endoscopy in a Human Body
}

\author{
G. Vermeeren, E. Tanghe, A. Thielens, L. Martens, Member, IEEE, and W. Joseph, Member, IEEE
}

\begin{abstract}
Physical-layer characterization is important for design of in-to-out body communication for wireless body area networks (WBANs). This paper numerically investigates the path loss of an in-to-out body radio frequency (RF) wireless link between an endoscopy capsule and a receiver outside the body using a 3D electromagnetic solver. A spiral antenna in the endoscopy capsule is tuned to operate in the Medical Implant Communication Service (MICS) band at 402 MHz, accounting for the properties of the human body. The influence of misalignment, rotation of the capsule, and human body model are investigated. Semi-empirical path loss models for various homogeneous tissues and 3D realistic human body models are provided for manufacturers to evaluate the performance of into-out-body WBAN systems.
\end{abstract}

\section{INTRODUCTION}

A wireless body area network (WBAN) connects nodes that are situated on or in the body of a person. Applications of WBANs include medicine and health care, sports, and multimedia. As it facilitates movement among users, it has brought about a revolutionary change in patient monitoring and healthcare facilities. Active implants placed within the human body lead to better and faster diagnosis, thus, improving the quality of life of the patient. The development of wireless capsule endoscopy (WCE) enables the examination of areas of the small intestine that cannot be seen by other types of endoscopy. The benefits of the WCE in terms of a better diagnosis are obvious. Also, the patient's comfort improves, as there are no wires or tubes involved in the procedure.

The characterization of the physical layer is an important step in the development of a WBAN. A lot of studies investigated on-body propagation [1-11]. Less literature is available on modeling of propagation loss within or in-to-out the human body [12-17] and often the focus is on $2.4 \mathrm{GHz}$. A path loss (PL) model for in-body wireless implants (biocompatible implantable antennas) is proposed in [12]. Scenarios for channel modeling for an endoscopy application are proposed in [14]. In [13] and [18] similar investigations were presented, but both of them proposed the $2.4 \mathrm{GHz}$ frequency band, which led to a smaller antenna. The influence of different tissues at various frequencies is characterized for an in-to-out channel in [19], but the antenna design was not specified. A multi-implant scenario at $2.4 \mathrm{GHz}$ is investigated in [20] using insulated dipole antennas for specific locations such as the liver, heart, spleen, and the kidneys. A spiral antenna at $450 \mathrm{MHz}$ for ingestible capsule endoscope systems is designed in [21].

The authors are with iMinds / UGent-INTEC-WAVES, Ghent, Belgium (corresponding author is G. Vermeeren: +32 9 3314918; e-mail: gunter.vermeeren@intec.ugent.be).
The objective of this study is to investigate numerically the PL of an in-to-out body radio frequency (RF) wireless link between a wireless endoscopy capsule and a half wavelength dipole (reference receiver) outside the body using a 3D electromagnetic solver, applying the finite difference time domain (FDTD) method. This study focusses on the 402 to $405 \mathrm{MHz}$ Medical Implant Communication Service (MICS) band [22]. The MICS band is a lower power frequency band, with less risk to encounter interferences, and allows longer communication links because the attenuation is lower. A disadvantage is that the size of the antennas increases. According to the authors' knowledge, there is only a small amount of studies about in-to-out body propagation for this band. We selected the spiral antenna for wireless capsule endoscopy operating at $450 \mathrm{MHz}$ proposed by [21] and tuned it to operate at $402 \mathrm{MHz}$ by changing its dimensions. We investigated the path loss for the in-to-out channel for homogeneous and heterogeneous tissues of a child and adult human body model.

The analysis of this research can be used by manufacturers to evaluate the performance of in-body to outbody WBAN systems (in terms of link budget to better estimate the energy consumption of the wireless endoscopy capsule (in terms of battery life time). Another application of the presented models is cross-layer design to evaluate communication protocols for WBANs and energy consumption in short-range wireless networks [6].

\section{METHOD}

\section{A. Configurations}

Homogeneous tissues: The path loss is investigated for three different tissues at $402 \mathrm{MHz}$ : muscle tissue (permittivity $\varepsilon_{\mathrm{r}}=57.1$, conductivity $\sigma=0.8 \mathrm{~S} / \mathrm{m}$ ), skin tissue $\left(\varepsilon_{\mathrm{r}}=46.7, \sigma=0.69 \mathrm{~S} / \mathrm{m}\right)$, and esophagus tissue $\left(\varepsilon_{\mathrm{r}}=\right.$ $67.5, \sigma=1 \mathrm{~S} / \mathrm{m})$ are considered. These represent relevant tissues for the endoscopy configuration [14]. Aligned and misaligned configurations and rotation of the transmitting spiral antenna in the capsule are investigated. Figure 1 shows the investigated configuration.

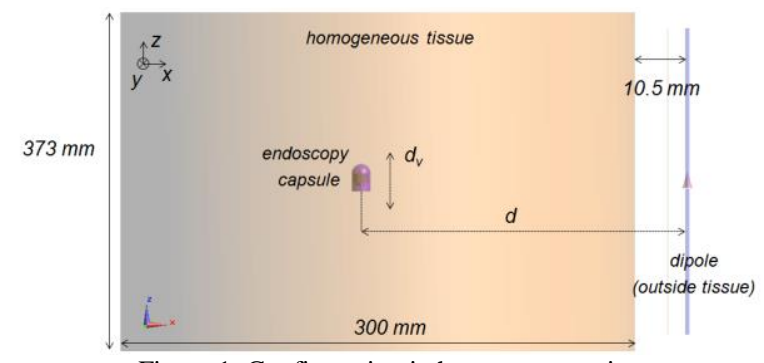

Figure 1: Configuration in homogeneous tissue. 
Heterogeneous phantom models: Two heterogeneous human body models from the Virtual Population [23] are investigated: a 6-year old boy (denoted Thelonious), and an adult man (denoted Duke). The Virtual Population models are based on magnetic resonance images (MRI) of healthy volunteers. The tissue properties for the frequency of $402 \mathrm{MHz}$ are assigned from Gabriel's database [24]. The PL at $402 \mathrm{MHz}$ is investigated in these models for positions inside the digestive tract, as shown in Figure 2, and compared with those in homogeneous tissues. The free-space dipole is positioned outside the body and is evaluated for two configurations: the free-space dipole is situated in a fixed position at a distance of $18.5 \mathrm{~cm}$ from the body next to the arm in the case of the adult man (Figure 2a) and at $13.5 \mathrm{~cm}$ in front of the body for the child model, at the height of the large intestine (Figure $2 b)$. Positions along the tract are selected with $x_{-}, y_{-}, z_{-}$ separations of $20 \mathrm{~mm}$ or $22 \mathrm{~mm}$.

Out-body antenna: We selected a half wavelength dipole antenna for the receiving antenna outside the body. The

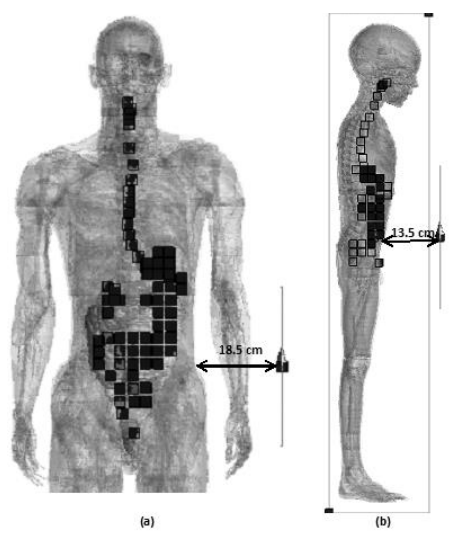

Figure 2: Configuration in heterogeneous human body models: (a) Duke, and (b) Thelonious.

free-space dipole is modeled and tuned to resonate at 402 $\mathrm{MHz}$. The thickness of the perfect electric conductor (PEC) structures is set at $2 \mathrm{~mm}$ and the gap is $1 \mathrm{~mm}$.

In-body antenna: We selected the spiral antenna of [21], designed for a wireless endoscopy capsule, as transmitting

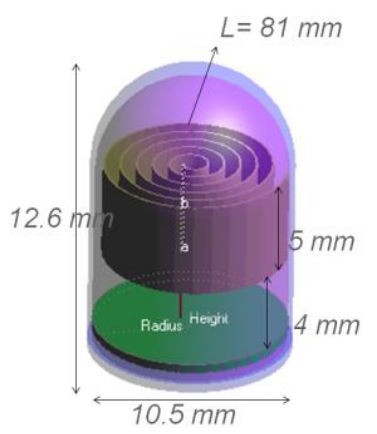

Figure 3: Wireless endoscopy capsule with spiral antenna of [21].

antenna inside the body and tuned it to $402 \mathrm{MHz}$. The antenna is composed of a spiral PEC structure and a ground plane separated at a certain distance to place the source in between (see Figure four of [21]). The shell of the capsule consists of a dielectric (Ultem material, $\varepsilon_{\mathrm{r}}=$ $3.15, \sigma=0 \mathrm{~S} / \mathrm{m})$. Figure 3 shows the dimensions of the capsule and antenna.. We remark that the antenna is not a very good radiator as the minimum reflection coefficient varies between $-7 \mathrm{~dB}$ (in muscle tissue) and $-5.5 \mathrm{~dB}$ (in esophagus tissue).

FDTD solver: We used the 3D FDTD solver available in SEMCAD-X (SPEAG, Switzerland) for performing the simulations. The simulation settings are the same in all the cases: harmonic simulations at $402 \mathrm{MHz}$ are run with a simulation time of 14 periods; uniaxial perfectly matched layer (UPML) boundary conditions terminate the simulation domain and absorb at least $95 \%$ of the incident power; the padding is set to $\lambda / 4$; and, a maximum grid step of $2 \mathrm{~mm}$ is applied inside the body tissues.

\section{PATH LOSS IN HOMOGENEOUS TISSUES}

This section discusses the in-to-out body propagation and path loss for homogeneous tissues. PL models are presented and the influence of misalignment, rotation in the body, and the absorption are determined. We analyze first homogeneous tissues because in this paper we aim at comparing the propagation in homogeneous tissues with the heterogeneous models. In contrary to layered tissues, homogeneous tissues can be replaced by liquids in practical setups to evaluate the performance of wireless endoscopy capsules and systems of manufacturers.

\section{A. Analysis of in-to-out body propagation}

$\mathrm{PL}$ is defined as the ratio of the input power at port $1\left(\mathrm{P}_{\text {in }}\right)$ to the power received at port $2\left(\mathrm{P}_{\text {rec }}\right)$ in a two-port setup. $\mathrm{PL}$ in terms of transmission coefficient is defined as $1 /\left|S_{21}\right|^{2}$ with respect to $50 \Omega$.

$$
\left.P L\right|_{\mathrm{dB}}=\left(P_{\mathrm{in}} / P_{\mathrm{rec}}\right)=-10 \log _{10}\left(\left|S_{21}\right|^{2}\right)=-\left|S_{21}\right|_{\mathrm{dB}} .
$$

This allows us to regard the setup as a two-port circuit for which we determine $\left|\mathrm{S}_{21}\right|$ (in $\mathrm{dB}$ ) with reference impedances of $50 \Omega$ at both ports. To model the path loss between the transmitting and the receiving antenna as a function of the distance, we use the following semi-empirical formula, expressed in $\mathrm{dB}$ and based on the Friis formula [25]:

$$
P L_{\mathrm{dB}}(\mathrm{d})=P L_{0, \mathrm{~dB}}+10 n \log _{10}\left(d / d_{0}\right)=-\left|S_{21}\right|_{\mathrm{dB}}
$$

where the total antenna separation $d$ is expressed in millimeter in this paper, $\mathrm{PL}_{0, \mathrm{~dB}}$ is the path loss in decibel at a reference distance $\mathrm{d}_{0}(250 \mathrm{~mm}$ in this paper), and $\mathrm{n}[-]$ is the path loss exponent, which equals to 2 in the free space. The antenna is included as a part of the channel model throughout this analysis $[6,13,18,20,27]$.

\section{B. Path loss parameters in homogeneous tissue}

Table 1 summarizes the parameters of the PL models for homogeneous tissues in this paper, obtained using Equation 2 and fitting the parameters $P L_{0, \mathrm{~dB}}$ and $n$ to the simulation data. The path loss exponents in the tissues are similar and about equal to 3 . The homogeneous esophagus tissue has a higher path loss constant $P L_{0, \mathrm{~dB}}(61 \mathrm{~dB}$ compared to 56 to $57 \mathrm{~dB})$ 
Table 1: Parameters for path lossmodels for homogeneous and heterogeneous tissues and phantoms $(\mathrm{d} 0=250 \mathrm{~mm})$.

\begin{tabular}{|c|c|c|c|c|c|}
\hline Tissue type & Configuration & PL0 (dB) & $n$ value & $\sigma$ value & $R 2$ value \\
\hline \multirow[t]{4}{*}{ Homogeneous } & Muscle, aligned antennas & 57.69 & 3.17 & 0.18 & 0.99 \\
\hline & Skin, aligned antennas & 56.81 & 3.00 & 0.16 & 0.99 \\
\hline & Esophagus, aligned antennas & 61.33 & 3.05 & 0.15 & 0.99 \\
\hline & Esophagus, misaligned antennas & 69.22 & 6.93 & 1.24 & 0.98 \\
\hline \multirow[t]{2}{*}{ Heterogeneous } & Thelonius (child) & 49.77 & 7.91 & 1.97 & 0.75 \\
\hline & Duke (adult) & 56.83 & 7.10 & 2.99 & 0.70 \\
\hline
\end{tabular}

due to the higher conductivity compared to the muscle and skin tissues. The $R^{2}$ values (coefficient of determination) are about 0.99 , indicating the linear relationship between $P L$ in decibel and distance, as these models are deterministic.

\section{Influence of antenna misalignment}

To study the influence of the misalignment, the antennas are shifted vertically. First, an antenna separation of $160.5 \mathrm{~mm}$ (spiral is positioned $150 \mathrm{~mm}$ from the tissue boundary, see Figure 1) is selected and the spiral is shifted vertically with a vertical displacement $\mathrm{dv}$ from $-100 \mathrm{~mm}$ to $+100 \mathrm{~mm}$. Figure 4a shows the PL for vertical displacement $\mathrm{dv}$ of the spiral. The curves are almost symmetric and the small disagreement is due to the spiral's non symmetric form. The PL increases by $3.3 \mathrm{~dB}$, considering the same tissue when the spiral is shifted at $100 \mathrm{~mm}$. The highest PL occurs in the esophagus tissue due to the highest conductivity. For larger separations d ( 250 to $360 \mathrm{~mm}$ ), misalignment between antennas causes the in-to-out body link to experience 10 to 12 $\mathrm{dB}$ differences for the same distance. Table 1 shows the influence of misalignment on the PL models: misalignment between antennas increases the path loss exponent to more than the double of when the antennas are aligned ( $n=3$ to 6.9 when misaligned in esophagus). Moreover the PL constant $P L_{0, \mathrm{~dB}}$ increases about $7 \mathrm{~dB}$ when misaligned $(69 \mathrm{~dB}$ compared to $61 \mathrm{~dB}$ for esophagus in Table 1).

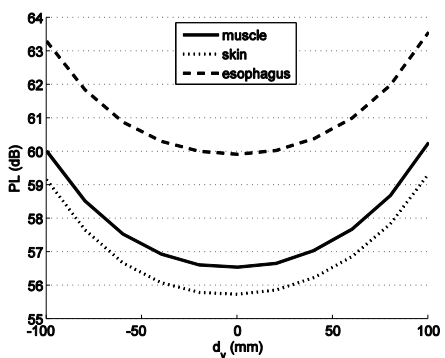

(a)

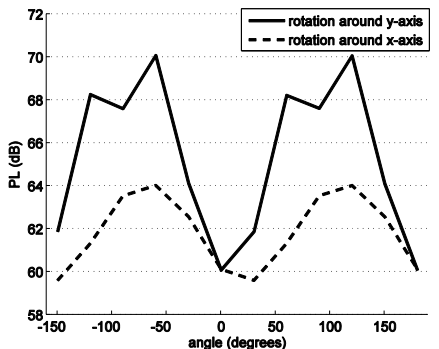

(b)

Figure 4: Influence of (a) vertical misalignment and (b) rotation of endoscopy capsule on path loss, PL in homogeneous tissue.

\section{Influence of antenna rotation}

The evaluation is performed for the esophagus tissue because the losses are the highest. Since the spiral is nearly symmetric, the influence of rotation around the z-axis is negligible. Only rotation around $\mathrm{x}$-axis and $\mathrm{y}$-axis is investigated. Figure $4 \mathrm{~b}$ shows the PL when the spiral is rotated around the $x$ - and $y$-axis, respectively. The figure is symmetrical because the position of the spiral with respect to the dipole is the same when it is rotated for positive and negative angles. When the rotation angle is $90^{\circ}$ or $-90^{\circ}$, the PL is maximum, because the transmitter is completely perpendicular to the dipole and is not pointing to it (Figure 1). The minimum PL around $\mathrm{x}$-axis is obtained with a rotation angle of $30^{\circ}$. The variation on the PL $(\triangle P L)$ for rotation around the $\mathrm{x}$-axis is $4 \mathrm{~dB}$. Analog results are obtained when rotating around the $\mathrm{y}$-axis. The variation $\triangle P L$ for rotation around the $y$-axis is $10 \mathrm{~dB}$. The effect of the rotation around this axis is larger because the spiral changes from pointing to the dipole to pointing directly to the opposite direction. Also, the $y$ - and $z$-components of the electric field $E$ is higher than the $x$ component of $E$ (Figure 1 ), so the effect of the rotation around $y$ is larger.

\section{PATH LOSS FOR HETEROGENEOUS PHANTOM MODELS}

\section{A. Path loss models}

Figure 5 compares the simulated PL in two realistic human body models with the PL in homogeneous tissues for different separations. A comparison between PL results of the adult and obese adult man will be made further. Figure 5 shows that of course different PL values can occur at a certain transmitter $\mathrm{Tx}$ - receiver Rx separation for different locations throughout the digestive tract (crosses for adult and circles for child in Figure 5): The capsule travels in the heterogeneous models along a tract through the pharynx, the esophagus, the stomach lumen, and the small and large intestines' lumen. Moreover, misalignments between Tx and $\mathrm{Rx}$ antennas occur. In general, the PL increases with the

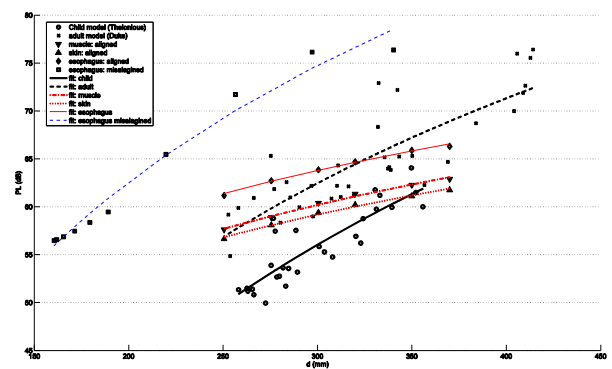

Figure 5: Path Loss, Pl in homogeneous and heterogeneous human body models. 
distance between antennas for heterogeneous tissues. The slope of simulated PL in the realistic human body models is clearly higher than for the PL in homogenous tissues due to the changes in the characteristic impedances of the different tissues and misalignment situations when traveling along the digestive tract.

Figure 5 shows that the path loss values for Duke (adult) are higher than the path loss values for Thelonious (child). This is due to the position of the dipole antenna (see Section 'Configurations') since for the child Thelonious it is placed in front of the body and for the adult Duke it is positioned at one side with the arm between the two antennas. The tissues in the arm add additional losses. Also the distance between body and dipole is smaller for Thelonious $(13.5 \mathrm{~cm})$ than for Duke $(18.5 \mathrm{~cm})$. The path loss for misalignment in the homogeneous esophagus tissue can be considered as the worst case. Table 1 summarizes for $n$ and $P L_{0}$ values obtained using linear regression fitting (Equation 2). The PL exponents for Thelonious and Duke are similar and about 7 to 8 in Table 1. The path loss in heterogeneous tissues is higher than in homogeneous tissues ( $n$ about 3 ) because of the influence of the surrounding tissues and the mixture of alignment and misalignment situations. The regression models for the heterogeneous phantoms have $R^{2}$ values (a coefficient of determination) of about 0.7 , which is good. An F-test concluded that the regression is significant, i.e., $\mathrm{n} \neq 0$, at the 5\% significance level $(F=78-81, p<10-9)$. Standard deviations of 2.0 and $3.0 \mathrm{~dB}$ for the child and adult model are obtained, respectively. Finally, Table 1 shows that larger standard deviations $\sigma$ correspond with lower $R^{2}$ values. These regression models can be used for link budget calculations for in-to-out body communications.

\section{CONCLUSIONS}

Path loss and absorption of an in-to-out body radio frequency (RF) wireless link between an endoscopy capsule and a receiver outside the body are numerically investigated. Three relevant homogeneous tissues (muscle tissue, skin tissue, and esophagus) and three heterogeneous human body models of an adult, obese adult, and a child are considered. A spiral antenna in the endoscopy capsule is tuned to operate in the MICS band at $402 \mathrm{MHz}$ accounting for the properties of the human body. The misalignment between antennas causes an increase of the path loss exponent from 3 to 7 and an additional shift of $7 \mathrm{~dB}$ in the esophagus tissue. An increase in the path loss due to the rotation of the antenna up to $10 \mathrm{~dB}$ is observed. Path loss models for different homogeneous tissues and two realistic human body models are provided, enabling manufacturers to evaluate the performance of inbody to out-body WBAN systems. Path loss exponents in heterogeneous models are about 7 to 8 and higher than for homogeneous tissues. In this paper two realistic heterogeneous phantom models are investigated. As there are many variations among people, future research should account for morphology.

\section{REFERENCES}

[1] G. O. Young, "Synthetic structure of industrial plastics (Book style with paper title and editor)," in Plastics, 2nd ed. vol. 3, J. Peters, Ed. New York: McGraw-Hill, 1964, pp. 15-64.
[2] W.-K. Chen, Linear Networks and Systems (Book style). Belmont, CA: Wadsworth, 1993, pp. 123-135.

[3] H. Poor, An Introduction to Signal Detection and Estimation. New York: Springer-Verlag, 1985, ch. 4.

[4] B. Smith, "An approach to graphs of linear forms (Unpublished work style)," unpublished.

[5] E. H. Miller, "A note on reflector arrays (Periodical style-Accepted for publication)," IEEE Trans. Antennas Propagat., to be published.

[6] J. Wang, "Fundamentals of erbium-doped fiber amplifiers arrays (Periodical style - Submitted for publication)," IEEE J. Quantum Electron., submitted for publication.

[7] C. J. Kaufman, Rocky Mountain Research Lab., Boulder, CO, private communication, May 1995.

[8] Y. Yorozu, M. Hirano, K. Oka, and Y. Tagawa, "Electron spectroscopy studies on magneto-optical media and plastic substrate interfaces(Translation Journals style)," IEEE Transl. J. Magn.Jpn. vol. 2, Aug. 1987, pp. 740-741 [Dig. $9^{\text {th }}$ Annu. Conf. Magnetics Japan, 1982, p. 301].

[9] M. Young, The Techincal Writers Handbook. Mill Valley, CA: University Science, 1989.

[10] J. U. Duncombe, "Infrared navigation-Part I: An assessment of feasibility (Periodical style)," IEEE Trans. Electron Devices, vol. ED11, pp. 34-39, Jan. 1959.

[11] S. Chen, B. Mulgrew, and P. M. Grant, "A clustering technique for digital communications channel equalization using radial basis function networks," IEEE Trans. Neural Networks, vol. 4, pp. 570578, July 1993

[12] R. W. Lucky, "Automatic equalization for digital communication," Bell Syst. Tech. J., vol. 44, no. 4, pp. 547-588, Apr. 1965.

[13] S. P. Bingulac, "On the compatibility of adaptive controllers (Published Conference Proceedings style)," in Proc. 4th Annu. Allerton Conf. Circuits and Systems Theory, New York, 1994, pp. 8 16.

[14] G. R. Faulhaber, "Design of service systems with priority reservation," in Conf. Rec. 1995 IEEE Int. Conf. Communications, pp. 3-8.

[15] W. D. Doyle, "Magnetization reversal in films with biaxial anisotropy," in 1987 Proc. INTERMAG Conf., pp. 2.2-1-2.2-6.

[16] G. W. Juette and L. E. Zeffanella, "Radio noise currents n short sections on bundle conductors (Presented Conference Paper style)," presented at the IEEE Summer power Meeting, Dallas, TX, June 2227, 1990, Paper 90 SM 690-0 PWRS

[17] J. G. Kreifeldt, "An analysis of surface-detected EMG as an amplitude-modulated noise," presented at the 1989 Int. Conf Medicine and Biological Engineering, Chicago, IL.

[18] J. Williams, "Narrow-band analyzer (Thesis or Dissertation style)," Ph.D. dissertation, Dept. Elect. Eng., Harvard Univ., Cambridge, MA, 1993.

[19] N. Kawasaki, "Parametric study of thermal and chemical non equilibrium nozzle flow," M.S. thesis, Dept. Electron. Eng., Osaka Univ., Osaka, Japan, 1993.

[20] J. P. Wilkinson, "Nonlinear resonant circuit devices (Patent style)," U.S. Patent 3624 12, July 16, 1990.

[21] SH Lee, J Lee, YJ Yoon, SPC Cheon, K Kim, S Nam, A wideband spiral antenna for ingestible capsule endoscope systems: experimental results in a human phantom and a pig. IEEE Trans. Biomed. Eng. 58(6), 1734-1741 (2011)

[22] ETSI, ETSI EN 301 839-1 V1.3.1 (2009-10). Electromagnetic compatibility and radio spectrum matters (ERM); short range devices (SRD); ultra low power active medical implants (ULP-AMI) and peripherals (ULP-AMI-P) operating in the frequency range $402 \mathrm{MHz}$ to $405 \mathrm{MHz}$; part 1: technical characteristics and test methods. (2009). Accessed 28 January 2014

[23] A Christ, W Kainz, E Hahn, K Honegger, M Zefferer, E Neufeld, W Rascher, R Janka, WBautz, J Chen, B Kiefer, P Schmitt, H Hollenbach, J Shen, M Oberle, D Szczerba, A Kam, J Guag, N Kuster, The virtual family-development of surface-based anatomical models of two adults and two children for dosimetric simulation. Phys Med. Biol. 55(2), 23-38 (2010)

[24] S Gabriel, RW Lau, C Gabriel, The dielectric properties of biological tissues: measurements in the frequency range $10 \mathrm{~Hz}$ to $20 \mathrm{GHz}$. Phys. Med. Biol. 41(11), 2251-2269 (1996)

[25] CA Balanis, Antenna Theory, Analysis and Design, 1st ed. (Harper \& Row, New York, 1982), pp. 64-65 